\title{
Distribution of Testudo graeca in the western Mediterranean according to climatic factors
}

\author{
José Daniel Anadón ${ }^{1,2, *}$, Andrés Giménez ${ }^{1}$, Eva Graciá ${ }^{1}$, Irene Pérez ${ }^{1}$, Marcos Ferrández ${ }^{3}$, \\ Soumia Fahd ${ }^{4}$, Hassan El Mouden ${ }^{5}$, Mohsen Kalboussi ${ }^{6}$, Tarek Jdeidi ${ }^{7}$, Said Larbes ${ }^{8}$, \\ Rachid Rouag ${ }^{9}$, Tahar Slimani ${ }^{5}$, Mohammed Znari ${ }^{10}$, Uwe Fritz $^{11}$
}

\begin{abstract}
Despite being one of the most charismatic elements of the Mediterranean Basin fauna and its threatened status, the western Mediterranean range of Testudo graeca is at present very poorly known. The present work provides the most detailed geographical and ecological description for the North African clade of $T$. graeca so far. We gathered 283 occurrence data of T. graeca in North Africa and modelled the distribution by means of presence-only distribution modelling tools. The obtained model was then projected to southern Europe in order to explore whether the environmental characteristics of European populations fall into the predicted niche of the species in North Africa.

T. graeca showed a wide environmental range in North Africa. Presence localities ranged from the sea level to $2090 \mathrm{~m}$ of altitude and from 116 to $1093 \mathrm{~mm}$ of annual precipitation. The presence-only model indicates that distribution in North Africa is mainly related to rainfall, specifically rainfall values in the wettest and coldest quarter of the year. The distribution model showed a range of ca. $1000000 \mathrm{~km}^{2}$. The projection of the model to southern Europe showed that the southern Iberian and Balkan Peninsulas, as well as most Mediterranean islands, present climatic conditions within those found in the range of the species in North Africa.
\end{abstract}

Keywords: ecological niche modelling, limiting factors, Maxent, North Africa, species distribution modelling, spur-thighed tortoise.

\section{Introduction}

1 - Área de Ecología, Dept. de Biología Aplicada, Universidad Miguel Hernández, Elche, Alicante, Spain

2 - Dept. of Ecological Modelling, UFZ - Helmholtz Center for Environmental Research, Leipzig, Germany

3 - Centro de Recuperación de Fauna de Santa Faz, Alicante, Spain

4 - Département de Biologie, Faculté des Sciences, Université Abdelmalek Essaâdi, BP. 2121, Tétouan, Morocco

5 - Laboratoire d'Ecologie Animale Terrestre, Faculté des Sciences, Semlalia, Université Cadi Ayyad, BP 2390, 40000 Marrakech, Morocco

6 - Institut Sylvo-Pastoral, Jendouba, 8110 Tabarka, Tunisia

7 - Zoology Department, Science Faculty, Alfateh University, Tripoli, Libya

8 - Faculté des Sciences Biologiques et Agronomiques, Université M. Mammeri, Tizi-Ouzou, Algeria

9 - Centre Universitaire d'El Tarf, 36100 El Tarf, Algeria

10 - Laboratory of Biodiversity \& Ecosystem Dynamics, Dept. of Biology, Faculty of Science, Semlalia, Cadi Ayyad University, BP 2390, 40000 Marrakech, Morocco

11 - Museum of Zoology (Museum für Tierkunde), Senckenberg Dresden, A. B. Meyer Building, D-01109 Dresden, Germany

*Corresponding author; e-mail: jdanadon@gmail.com. Present address: Dept. de Biología de la Conservación,
The spur-thighed tortoise Testudo graeca (L., 1758) is one the most charismatic species of the circum-Mediterranean fauna. In the western Mediterranean, T. graeca has its core range in North Africa with small and isolated European populations in southern Spain and several Mediterranean islands. The species is considered endangered across its entire range (classified as vulnerable; IUCN, 2002) and the main threats are habitat loss (IUCN, 2002) and collection for the pet trade (Lambert, 1979; Pérez et al., 2004; Pérez et al., 2011).

Despite its charisma and endangered status, the range of $T$. graeca in North Africa is at present poorly known; even an ecological and a detailed geographical description of the whole range of the species is lacking. Regarding distribution, published knowledge is diffi-

Estación Biológica de Doñana CSIC, Av. Americo Vespucio s/n, 41092 La Cartuja, Sevilla, Spain 
cult to access due to high bibliographic dispersion. Only for Morocco the distribution has been previously summarized in some detail, using raw occurrence data (Lambert, 1983; Bons and Geniez, 1996; see also Schleich, Kästle and Kabisch, 1996). For the remaining countries of North Africa, the range is only superficially known, being described as a wide strip along the coast in Algeria and Tunisia with a small isolated area in the Cyrenaica Peninsula (Libya). In the same vein, the environmental factors controlling the distribution of the species in North Africa at a large scale have only been tackled for Morocco in the pioneering work of Lambert (1983) and in more depth for southeast Spain (Anadón et al., 2006, 2007, 2010). These studies indicate a meridional limit of the species related to rainfall (aridity) and a septentrional (or altitudinal) limit related to minimal extreme temperatures.

In contrast to large scale distribution and species-habitat relationships, phylogeography of North African populations of T. graeca has recently received notable attention (Álvarez et al., 2000; Fritz et al., 2007, 2009; Graciá et al., 2011; Salinas et al., 2011). Fritz et al. (2007, 2009) showed that $T$. graeca comprises six major genetic clades, that diverged 4.2 to $1.8 \mathrm{Ma}$ ago; five of them from the Caucasus region and the Middle East, while the sixth clade corresponds to the western Mediterranean populations. Within the western Mediterranean clade, a finer structure of more recent origin (1.7 to $0.4 \mathrm{Ma}$ ago) has been identified that matches well with previously morphologically defined subspecies (Fritz et al., 2009). In this sense, the North African populations of T. graeca, the subject of this work, constitute a well-defined genetic unit.

Genetic investigations also revealed that the western European populations of $T$. graeca (Spain and islands of Mallorca, Sicily and Sardinia) belong to North African lineages (Fritz et al., 2009; Salinas et al., 2011; Vamberger et al., 2011). This suggests a recent origin for these populations due to natural or human-mediated dispersal in historic or prehistoric times. In the present work, we determine whether the environmental characteristics of western European populations fall into the niche of the species in North Africa, and explore the possible environmental factors limiting its European range.

In summary, our objectives are: (i) to describe the ecological and geographical range of T. graeca in North Africa, (ii) to build a distribution model of the species in North Africa, and (iii) to explore whether the environmental characteristics of southwestern European populations fall into the niche of the species in North Africa.

\section{Methods}

\section{Data collection and niche characterization}

We gathered 283 records of T. graeca from North Africa (204 from Morocco, 58 from Algeria, 12 from Tunisia, and 9 from Libya). The bulk of the records ( $n=174 ; 61 \%$ ) were obtained from field-work conducted in the study area by the authors during the last decade. Present records may not accurately reflect the climatic niche and range of the species because the species may at the present time be absent in climatically suitable areas due to human-related extinction processes. Thus, our database also included the locality records $(n=109)$ provided by Lambert (1983) to more accurately reflect the climatic range of the species. As a precautionary measure, historical records of the species in Egypt were not considered due to possible misidentification with T. kleinmanni.

In order to obtain a first description of the niche of the species, occurrence data were characterized in relation to basic climatic descriptors (mean annual precipitation and temperature) plus the mean minimum temperature of the coldest month, that has been indicated as an important limiting factor in the species (Lambert, 1983; Anadón et al., 2006). Climatic variables were obtained from WorldClim. (Hijmans et al., 2005, http://www.worldclim.org). Both bioclimatic variables, originally at a resolution of $30^{\prime \prime}$, and tortoise records were transformed to a resolution of $5^{\prime}$ that constitutes the resolution of our work. Transformation of environmental variables was made by averaging the original values in the new $5^{\prime}$ cell. To better understand the environmental position of T. graeca population within Mediterranean climate, we calculated and represented the unmodified Emberger Index Qemb $=2000 \times P /\left(T^{2}-t^{2}\right)$, where $P$ is mean annual rainfall $(\mathrm{mm}), T$ is the mean maximum temperature of the warmest month and $t$ the mean minimum temperature of the coldest month (both measures in Kelvin). This index Qemb together with $t$ describes the main Mediterranean bioclimates (Emberger, 1930; Daget, 1977). 


\section{Distribution modelling}

A distribution model for the species in North Africa as well as its projection in southern Europe was assessed by means of Maxent 3.3.3 (Phillips et al., 2006, 2008). This software requires only presence data and performs well compared to other methods (Elith et al., 2006). Maxent applies a maximum entropy method to predict habitat suitability as a function of environmental variables. The distribution of species, and particularly ectotherms, is limited at a large scale by climatic factors (Guisan and Hofer, 2003; Kearney and Porter, 2004; Buckley et al., 2008; Jimenez-Valverde et al., 2011). For this reason we considered as environmental variables the 19 bioclimatic variables from WorldClim plus evapotranspiration and aridity indexes (http:// www.cgiar-csi.org/). All predictor variables were checked for collinearity by calculating all pair-wise Spearman's rank correlation coefficients. In a first round, we considered that a pair of predictor variables were strongly correlated when $\left|\mathbf{r}_{\mathrm{s}}\right|=0.7$ (e.g. Rhodes et al., 2009). Correlated variables were excluded iteratively, each turn excluding the correlated variable with the highest number of strong correlations with other variables. This approach yielded a set of 10 climatic variables, a number that allows for complex species-climate relationships (Svenning et al., 2008) and larger than typical climate data sets, that contains fewer variables (e.g. from 5 to 7 variables; Brito et al., 2009; Rodder et al., 2009; Jimenez-Valverde et al., 2011). In order to avoid possible overfitting of the model and reduced transferability (Peterson et al., 2007), we reduced the number of variables by decreasing the correlation threshold to 0.65 , yielding a final climatic set of seven variables, four related to temperature (isothermality, min temperature of coldest month, mean temperature of wettest quarter and mean temperature of driest quarter) and three to precipitation (precipitation of wettest quarter, precipitation of warmest quarter and precipitation of coldest quarter). Isothermality was defined as the mean diurnal range divided by the annual temperature range (see Hijmans et al., 2005 for further details).
A visual inspection of the occurrence data (fig. 1) showed a notable clustering at two scales. First, at a local scale there were many occurrences around some particular locations where tortoise populations are abundant and subject to intense field work (e.g., Agadir-Souss and Tanger-Tetuan in Morocco or Chief in Algeria). At a large scale, there were differences in the density of locations from $\mathrm{E}$ to $\mathrm{W}$, being maximum in Morocco, particularly the half North and minimum in Tunisia. These large scale differences likely reflect overall differences in the sampling effort carried out by the different research teams in their respective working areas (countries or part of countries). Following general good modelling practices (Elith et al., 2010), in order to assess the effect of these two data clustering processes in the modelling procedures, we first developed niche and distribution models with this originally clustered database. The obtained models, not shown in this work, resulted in predictions (habitat quality maps) strongly biased by the following two clustering patterns: i) high quality habitat areas only around main clusters (see above); ii) an E-W habitat quality gradient with overall low quality areas in Libya and Tunisia, intermediate in Algeria and high in Morocco. To avoid an overweighting of these areas and regions in the modelling (and the underlying violation of statistical independence of observations in species distribution models; Dormann et al., 2007), we followed two procedures. Clustering at a local scale was minimized by filtering the data and deleting presence data from clusters (Rodder et al., 2009). Clusters were identified by means of a hierarchical clustering tree of the presence localities, using the coordinates (latitude, longitude) as classification variables. Here, clusters were arbitrarily defined as those groups of localities with a similarity $>99.5 \%$. We then selected at random one presence datum from each cluster. This procedure yielded a final data set, employed for modelling, of 179 cells of presence (113 in Morocco, 48 in Algeria, 10 in Tunisia, and 8 in Libya). Regarding large-scale clustering, Maxent allows for entering bias maps in the modelling in order to account for different sampling and non-equilibrium bias (Elith et al., 2010,

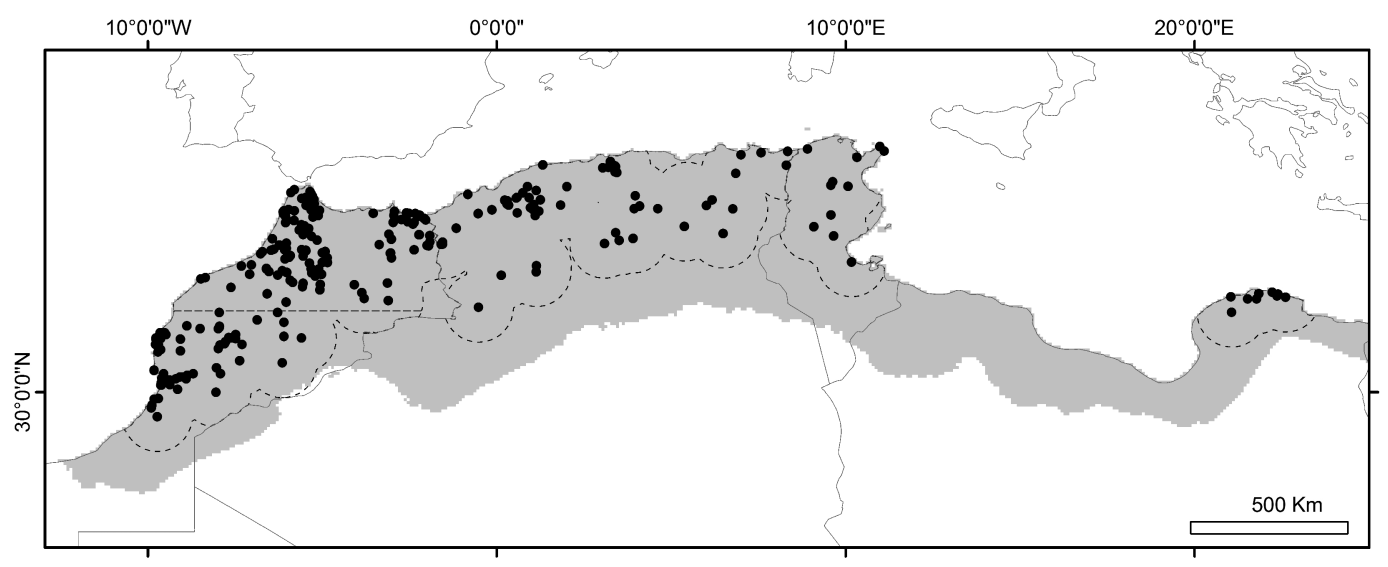

Figure 1. Locality records for Testudo graeca in North Africa $(n=283)$. Shaded area corresponds to the extent area employed for distribution modelling in North Africa. Inland continuous lines represent country boundaries. Dashed lines represent the buffer areas used for calculating weights for bias maps. 
Table 1. Calculation of the weights for the Maxent bias map. The weight of each zone was proportional to the density of locations (number of locations per unit area). Area of the zones expressed as number of $5^{\prime} \times 5^{\prime}$ cells. For a description of the area see Methods section.

\begin{tabular}{llcrcl}
\hline Zone & Description & No. locations & Area & Density & Weight \\
\hline 1 & S Morocco & 37 & 2285 & 0.0162 & 2.13 \\
2 & N Morocco & 76 & 2431 & 0.0313 & 4.11 \\
3 & Algeria & 48 & 4741 & 0.0101 & 1.32 \\
4 & Tunisia & 10 & 1306 & 0.0076 & 1 \\
5 & Libya & 8 & 507 & 0.0158 & 2.08 \\
\hline
\end{tabular}

2011). Thus, in order to minimize the bias effects due to differences in the presence data density at a regional level (increasing density from $\mathrm{E}$ to $\mathrm{W}$ ), we divided the study area into five zones and assigned a bias value to each zone proportional to its data density (see fig. 1 and table 1 ). The five zones corresponded to Algeria, Tunisia, and Libya, plus two zones in Morocco. Morocco was split into two zones at latitude $\mathrm{N} 32.30^{\circ}$ because density of occurrence data in the North was higher than in the South (likely reflecting different research groups and thus sampling intensities). When calculating the density of locations, using the total area of the countries or the zones inside the study area could have been misleading because each country contains a different proportion of unsuitable habitat (mainly the Saharan desert). Therefore, when calculating the density of locations in each zone, we used a buffer area of $1^{\circ}$ (aprox. $110 \mathrm{~km}$ ) around the presence locations (fig. 1). Although arbitrary, the zones defined by this buffer distance exclude the main areas unsuitable for the species in the south of the study area and thus our weight values reflect the density of locations in those areas where locations are more probable (table 1).

In order to avoid a subjective study area (modelling area) in North Africa we used for modelling that area in North Africa with less than $50 \mathrm{~mm}$ of annual rainfall. This area is biological meaningful since it includes all North Africa but leaving aside the most arid areas where the species does not occur (see fig. 1; minimum annual rainfall value of a location with presence data $=116 \mathrm{~mm}$, table 2). Maxent models were replicated 100 times with subsampling replication mode, each run leaving $20 \%$ of the presence data aside. The presented results are average values of these 100 model replicates. For every cell (in our case of $5^{\prime}$ edge length) of the study area, Maxent yields a value of probability for the presence of a given species, ranging from 0 to 1 . In order to quantify the distribution range of the species we also transformed these continuous probability values into three main distribution classes ('out of range', 'suboptimal' and 'optimal') defined by two threshold values $\mathrm{P}_{1}$ and $\mathrm{P}_{2}$. The 'out of range' area ranges between 0 and the lowest probability value predicted for any occurrence locality $\left(\mathrm{P}_{1}\right)$ and the species is not expected to be found in this area. The next probability class, ('suboptimal') ranges between $\mathrm{P}_{1}$ and the value that equals the sensibility and specifity of the model in relation to the test data set $\left(\mathrm{P}_{2}\right)$. This value is one of the most common thresholds employed in distribution habitat modelling to define species distribution ranges (Liu et
Table 2. Climate and altitude ranges for the study area (North Africa) and for the localities with occurrence of T. graeca. Minimum and maximum values for occurrence localities were obtained from all localities $(\mathrm{N}=283)$; median values were obtained from cluster-corrected data set ( $n=179)$ in order to diminish overweighting of intensively sampled areas. Temperatures are given in ${ }^{\circ} \mathrm{C}$; precipitations, in mm; altitude in m.a.s.l.

\begin{tabular}{lrrrrrrr}
\hline & \multicolumn{2}{c}{ North Africa } & & \multicolumn{3}{c}{ Occurrences } \\
\cline { 7 - 7 } & Min. & Max. & & Min. & Max. & Median \\
\hline $\begin{array}{l}\text { Annual mean } \\
\quad \text { temperature }\end{array}$ & 4.8 & 24.8 & & 9.9 & 21.7 & 17.0 \\
$\begin{array}{l}\text { Min. temperature } \\
\text { of coldest month }\end{array}$ & -10.8 & 13.4 & & -6.8 & 10.4 & 4.2 \\
$\begin{array}{l}\text { Annual precipitation } \\
\text { Altitude }\end{array}$ & $<1$ & 1292 & & 116 & 1092 & 406 \\
\hline
\end{tabular}

al., 2005). Suboptimal areas are usually statistically considered not part of the range but the presence of the species is very low though possible. The optimal habitat comprises all those cells with probability of presence $>P_{2}$. For representation purposes we further divided the optimal habitat in three classes classes (low, medium, high) that were obtained by dividing the remaining species probability range (probability of presence $>\mathrm{P}_{2}$ ) in three classes of the same length $\left(\mathrm{M}-\mathrm{P}_{2}\right) / 3$, where $\mathrm{M}$ is the maximum value of probability assigned to a cell.

The importance of each bioclimatic variable in explaining the observed distribution of T. graeca was evaluated by the percent contribution (PC) and permutation importance (PI) as assessed by Maxent. To determine PC, in each iteration of the training algorithm, the increase in regularized gain is added to the contribution of the corresponding variable, or subtracted from it if the change to the absolute value of lambda is negative. For PI, for each environmental variable in turn, the values of that variable on training presence and background data are randomly permuted. The model is reevaluated on the permuted data, and the resulting drop in training AUC is shown in the table, normalized to percentages. The exploration of the response curves obtained from Maxent were also used to complete the description of the climatic niche of the species.

Projection of the Maxent model obtained in North Africa was applied to Southern Europe (eastern limit: $26^{\circ} \mathrm{E}$, northern limit: $45^{\circ} \mathrm{N}$ ). When projecting the model we excluded those areas of Southern Europe which environmental characteristics are not present in the training area (study area in North Africa), as assessed by the MESS tool in Maxent.

\section{Results}

Niche of Testudo graeca in North Africa

Occurrence data showed a wide environmental range, with presence localities from the sea level to $2090 \mathrm{~m}$ of altitude (High Atlas, Mo- 


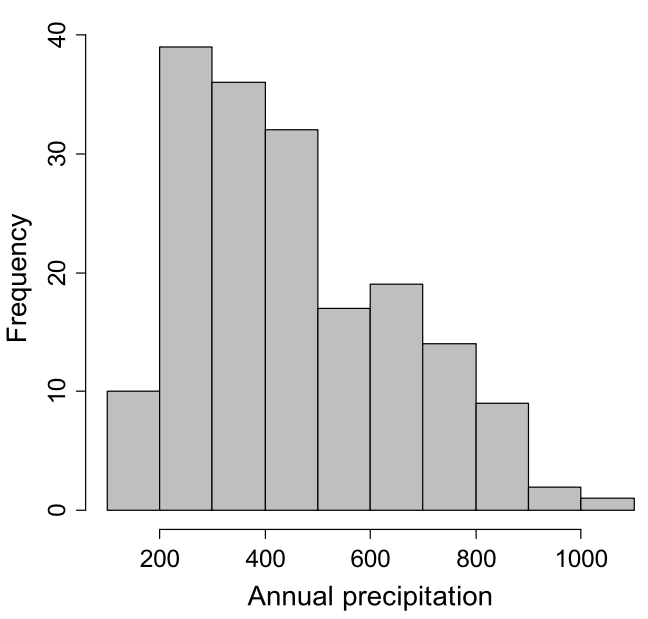

(A)

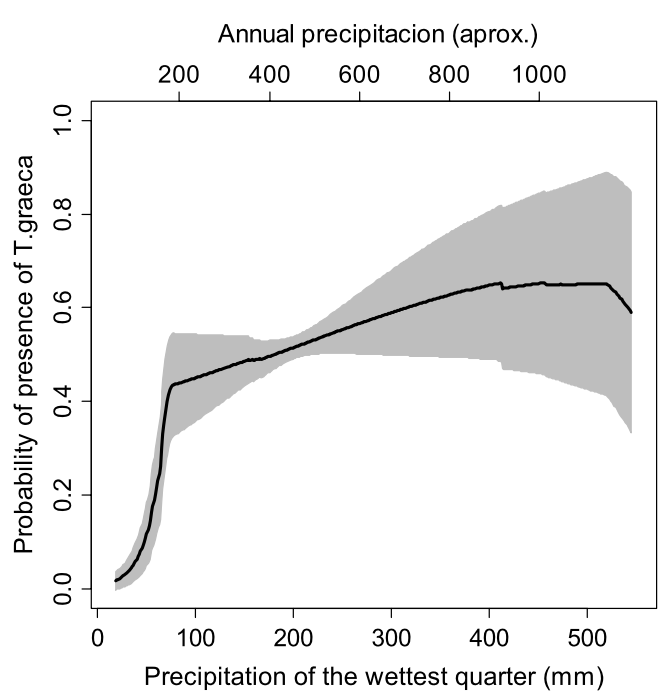

(B)

Figure 2. (A) Distribution of the locality records for Testudo graeca in relation to mean annual precipitation. Only cluster-corrected data were used $(n=179)$ to diminish overweighting of small-scale oversampled areas. (B) Response of $T$. graeca to precipitation of wettest quarter as assessed from Maxent. Shaded area represents the standard deviation from the 100 model replicates. The Y-axis on the top represents the approximate annual precipitation values corresponding to the precipitation of the wettest quarter values, as assessed from a regression model (see Discussion).

rocco) and from $116 \mathrm{~mm}$ (El Feidh, Algeria) of annual rainfall to $1093 \mathrm{~mm}$ (Tabarka, Tunisia; table 2). The distribution of presence localities in relation to rainfall has a median of $400 \mathrm{~mm}$ (fig. 2: top). As shown in fig. 3, T. graeca oc-

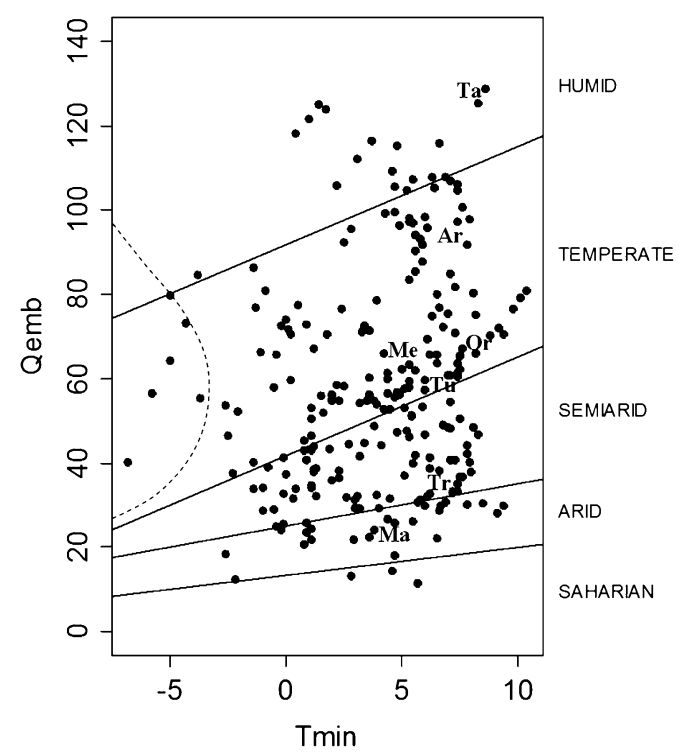

Figure 3. Environmental situation of the presence localities $(n=283)$ of Testudo graeca in North Africa in relation to the main Mediterranean bioclimates, as defined by the Emberger Index (Qemb) and minimum temperatures of the coldest month. Continuous lines separate main bioclimates. Dashed line defines High Mountain Mediterranean bioclimate. $\mathrm{Ar}=$ Alger, $\mathrm{Ma}=$ Marrakech, $\mathrm{Me}=$ Meknes, $\mathrm{Or}=$ Oran, $\mathrm{Ta}=$ Tangiers, $\mathrm{Tr}=$ Tripoli, $\mathrm{Tu}=$ Tunisia.

cupies all the main Mediterranean bioclimates from Saharian to Humid. Furthermore, four localities of T. graeca lie in the High Mountain Mediterranean climate.

Variables related to rainfall had a larger weight in the Maxent model than those related to temperature. In particular, the precipitation of wettest quarter had a weight ranging from 47-52\% depending of the metric employed (table 3 ). Among the rainfall variables, precipitation of the warmest quarter had consistently the lowest weight in all cases (8-10\%). Mean temperature of the driest quarter was the variable related to temperature with larger weight (8$11 \%)$ whereas the other temperature variables had generally marginal contributions $(<5 \%$; table 3 ). The response of $T$. graeca to precipitation of the wettest quarter (fig. 2) indicates that from 20 to $75 \mathrm{~mm}$ of precipitation (in the wettest queater) the presence of tortoises rapidly increases. Above $75 \mathrm{~mm}$ the probability of presence steadily increases at a lesser rate. 
Table 3. Relative constributions of the environmental variables to the Maxent models. Average and standard deviation of the 100 replicate models are shown. $\mathrm{PC}=$ Percent contribution; PI = Permutation importance. See Methods section for an explanation of PC and PI.

\begin{tabular}{lrc}
\hline & \multicolumn{2}{c}{ Climate model } \\
\cline { 2 - 3 } & \multicolumn{1}{c}{ PC } & PI \\
\hline $\begin{array}{l}\text { Isothermality } \\
\text { Min. temperature of }\end{array}$ & $4.1 \pm 1.6$ & $2.4 \pm 1.6$ \\
$\quad$ coldest month & $0.7 \pm 0.5$ & $1.8 \pm 1.5$ \\
$\quad$ Mean temperature of & $3.9 \pm 1.2$ & $8.8 \pm 2.2$ \\
$\quad$ wettest quarter & & \\
Mean temperature of & $7.6 \pm 2.3$ & $11.2 \pm 3.8$ \\
$\quad$ wettest quarter & & \\
Precipitation of wettest quarter & $47.4 \pm 6.5$ & $52.5 \pm 13.3$ \\
Precipitation of warmest quarter & $7.9 \pm 2.3$ & $10.4 \pm 3.3$ \\
Precipitation of coldest quarter & $28.4 \pm 6.9$ & $13 \pm 11.1$ \\
\hline
\end{tabular}

\section{Distribution of Testudo graeca in North Africa}

The obtained climatic model (fig. 4) covers most of Morocco, northern Algeria, Tunisia, and the Cyrenaica Peninsula (Libya). Threshold values used to define distribution classes ( $\mathrm{P}_{1}$ and $\mathrm{P}_{2}$, see Methods) were 0.0764 and 0.3471 , respectively. This threshold points suggest a total distribution area of approximately $1000000 \mathrm{~km}^{2}$, from which $600000 \mathrm{~km}^{2}$ are optimal (probability $>\mathrm{P}_{2}$ ) from a climatic perspective. The average AUC value of the model was 0.812 . The standard deviation values of the 100 replicates (fig. 4) can be considered low (mean value of the standard deviation across all cells for the climatic model $=0.03104$ ), indicating robust predictions. Major areas of uncertainty were located in Tunisia, particularly in coastal areas and in eastern Algeria. In general, the model follows the distribution of the occurrence locations, however it can be identified four areas for which the model predicts suitable climatic conditions but where the species is either rare or completely absent. These areas are the Middle Atlantic Plains and Plateau in Morocco, the Taza-Al Hoceima-Taunate region in the Rif Mountains (Morocco), the Bejaia-JjelSetif region in coastal Algeria and much of the Libyan coast.

As shown in fig. 4, in Morocco climatic suitability for the species is structured by the main mountain ranges. Most of the area north of the Anti-atlas, High Atlas, and Middle Atlas ranges (except the Middle Atlantic Plains and Plateau) is of medium to high climatic habitat quality. Climatic habitat quality is particularly high in the northernmost part of Morocco, including central and east (but not west) Riff Mountains, and the lower part of the watershed of the Moulouya River. In southern Morocco, a large area of high climatic habitat quality extends in the Atlantic coast from Agadir to Essaouira, including the low elevation foothills of the High Atlas. South of the Anti-Atlas, High Atlas, and Medium Atlas, the probability of occurrence drops considerably due to the strong aridity gradient. Climatic medium and high quality habitat occurs from Morocco to Algeria in the Tell Atlas, running parallel to the Mediterranean coast. The Saharan Atlas constitutes a medium climatic habitat quality patch for the species but is weakly connected to the main Algerian habitat patch (Tell Atlas). The Saharan Atlas is isolated from the Tell Atlas by the Salt Lakes (Algeria) and the High Plateau (AlgeriaMorocco), both with suboptimal climatic habitat quality. In the inland portion of eastern Algeria and Tunisia, the Aurés Mountains constitutes an area of lower habitat quality than the coastal areas. In Tunisia, high climatic habitat quality is therefore largely restricted to low-altitude areas in close proximity to the coast. The region around the Gulf of Gabes along the Tunisia coast presents mainly suboptimal climatic habitat quality. The range of the species continues from Tunisia to Libya with a narrow strip of suboptimal habitat quality (where no record of T. graeca is known). Medium to high quality habitat for T. graeca in the Cyrenaica Peninsula is restricted to the northern part, corresponding to a population isolated from the North African main range of $T$. graeca.

\section{Suitability of environmental conditions in Europe}

The projection of the model to southern Europe (fig. 5) shows that environmental condi- 

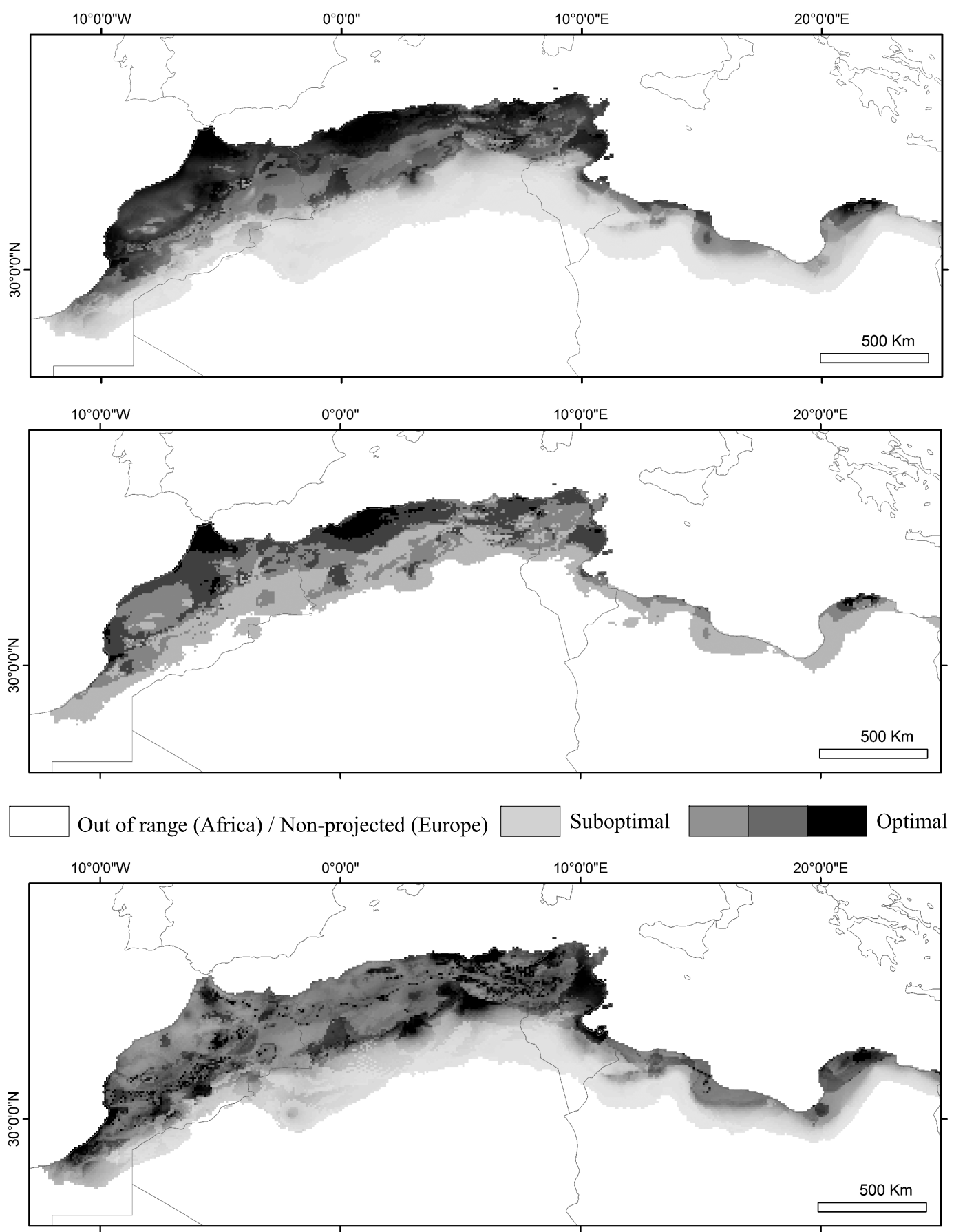

Figure 4. Top: Predicted probability of presence of Testudo graeca in North Africa according to climate variables, with darker shades indicating higher presence probability. Average prediction from 100 Maxent replicates. Center: distribution range for Testudo graeca in North Africa and habitat quality classes according to climatic variables. See Methods section for a definition of the classes of probability of presence of the species. Bottom: Standard deviation of the 100 Maxent replicates, for the predicted probability of presence of Testudo graeca in North Africa according to climatic variables. Darker shades indicates higher standard deviation values (range: $\min =0.0003$, $\max =0.1517$ ). In all cases, inland continuous lines represent country boundaries. 


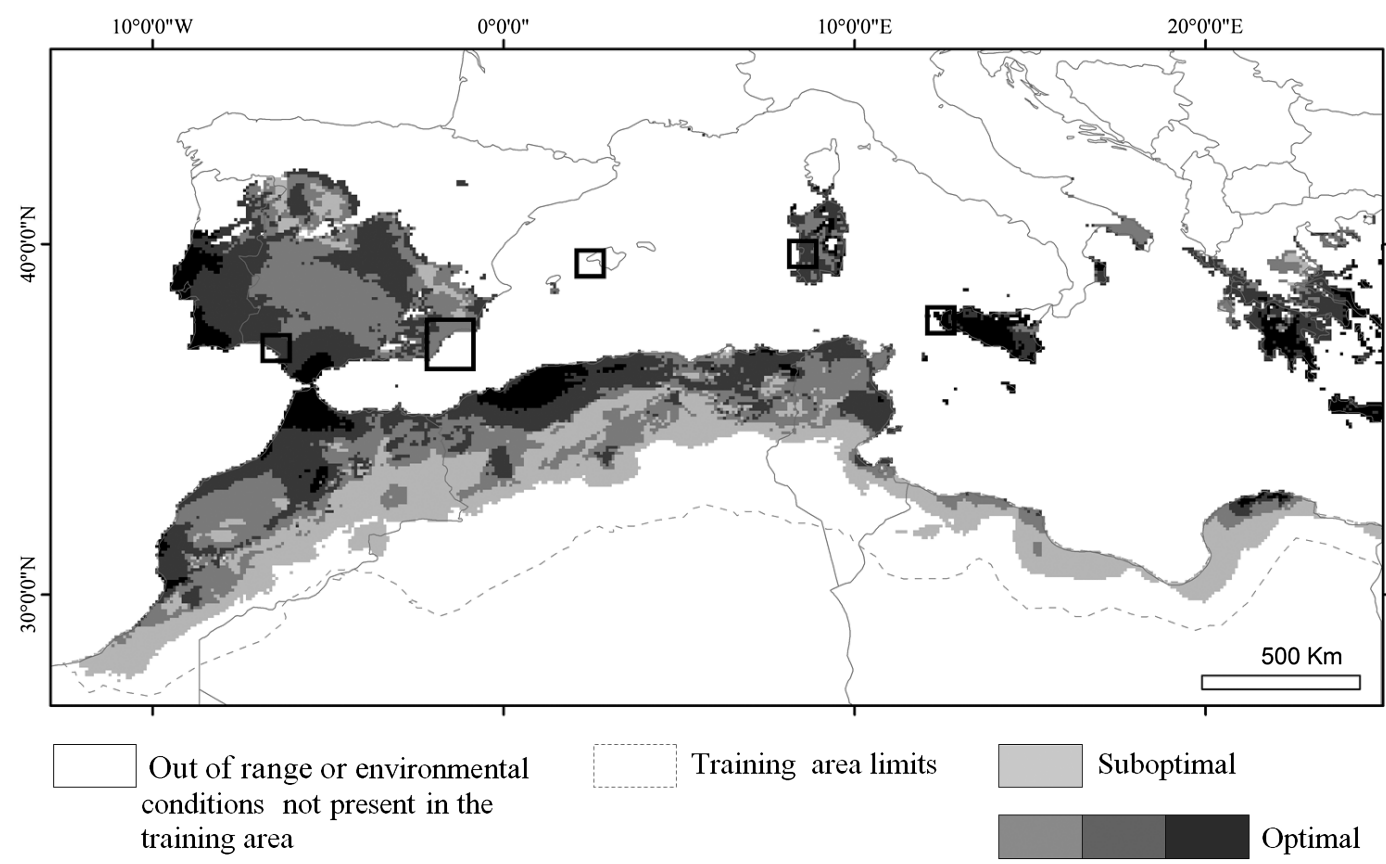

Figure 5. Predicted climatic habitat quality of Testudo graeca in southwestern Europe and North Africa based on the climatic distribution model obtained for North Africa. Black squares indicate reproductive western European populations. See Methods section for a definition of the classes of probability of presence of the species. The limits of the figure in Europe match the limits of the projected area (see Methods). Inland continuous lines represent country boundaries.

tions in Europe and even in some southernmost regions, like the Italian Peninsula and the northern part of the Balkan Peninsula, fall out of the environmental range occupied by the species in North Africa. The southern Iberian and Balkan Peninsulas, however, present environmental conditions within those of the range in North Africa. Regarding Mediterranean islands, Sardinia, Sicily, Cyprus and the small Aegian islands present suitable climatic habitat. Climatic conditions in Balearic islands and Corsica fall out of the North African climatic range of the species.

\section{Discussion}

The good knowledge of the distribution of a given species and the limiting environmental factors are basic requirements for developing adequate conservation strategies (Guisan and Thuiller, 2005). The present work provides the most detailed geographical and ecological - climatic - description of the North African clade of $T$. graeca range so far. In any case, it should be emphasized that our distribution model only relies on climatic variables and thus should be considered a description of the climatic potentiality for the species, and not of the current distribution.

Our results indicate that $T$. graeca occupies a very wide range of environmental conditions in North Africa, ranging from arid $(116 \mathrm{~mm}$ of annual rainfall) to very humid Mediterranean (1092 mm) environments, and being even present in high mountain niches (maximum altitude $=2090 \mathrm{~m}$ ). In fact, at a North African scale, the species is only limited by rainfall in the southern part of its range (i.e., Sahara). To have a more intuitive understanding of the response to rainfall we can translate the values of precipitation of the wettest quarter $\left(\mathrm{P}_{\mathrm{WQ}}\right)$ to values of annual precipitation $\left(\mathrm{P}_{\mathrm{A}}\right)$ (fig. 2). This is only possible due to the very high correlation between these two vari- 
ables $\left(\mathrm{r}_{\mathrm{s}}=0.97 ; \mathrm{P}_{\mathrm{A}}=18.95+2.1718 \times \mathrm{P}_{\mathrm{WQ}}\right)$. This conversion shows that the increase in the probability of presence of the species occurs from 60 to $180 \mathrm{~mm}$ of annual precipitation. It should be noted that the threshold value $\mathrm{P}_{2}$ used for separate suboptimal from optimal climatic habitat $\left(\mathrm{P}_{2}=0.3471 ; \mathrm{P}_{\mathrm{WQ}}=68 \mathrm{~mm} ; \mathrm{P}_{\mathrm{A}}=\right.$ $167 \mathrm{~mm}$ ) closely matches with the tipping point value of $180 \mathrm{~mm}$. Overall, the key role of rainfall is in agreement with previous descriptions of the niche of the species in Morocco (Lambert, 1983). However, our models did not detect a relevant limiting role of minimum extreme temperatures as earlier proposed (Lambert, 1983; Anadón et al., 2007). This does not imply that extreme minimum temperatures do not impose a strong physiological limit to the species, but rather that extreme minimum temperatures are likely to act as a limiting factor in only a few areas (mainly the High Atlas in Morocco) when considering North Africa as a whole.

The obtained climatic range of $T$. graeca in North Africa occupies roughly $1000000 \mathrm{~km}^{2}$. The range has been described in detail in the Results section, but some aspects deserve to be discussed here. The AUC value of the model indicates notable prediction ability (Manel et al., 2001), however, there are at least four areas for which the model predicts suitable climatic conditions, but where the species is either rare or completely absent. These areas of mismatch between model predictions and observed data likely point out different processes of ecological, biogeographical or conservation interest or concern. These areas are the Middle Atlantic Plains and Plateau in Morocco, the TazaAl Hoceima-Taunate region in the Rif Mountains (Morocco), the Bejaia-Jjel-Setif region in coastal Algeria and much of the Libyan coast. In the Atlantic and central Moroccan plains and plateau, a heavily human-populated region, tortoises became scarce in many areas due to collection for pet trade occurred in the past (Lambert et al., 1983). Additionally, it is likely that land use changes and habitat fragmentation may have increased the rarefaction of tor- toises, as occurred with other fauna (Cherkaoui et al., 2009). In any case, the climatic quality of some parts of this area, particularly in the south, seems to be also constrained by aridity, as also pointed out by Lambert (1983). Further research addressing the impact of land uses on the distribution of the species in North Africa is thus needed.

As for the other three areas, each without heavy environmental changes caused by land use, the absence of locality records could result from three factors: firstly, under-sampling in these areas could bias the current knowledge; secondly, local environmental factors could be responsible for limiting the presence of the species; and thirdly, the current distribution could reflect a pattern due to the biogeographical history of the species in North Africa. It has been proposed that the North African range of $T$. graeca experienced repeated expansion and contraction linked to Pleistocene and Holocene aridity cycles (Lambert, 1983; Fritz et al., 2009), and regions from where tortoises are presently absent may have been not recolonized yet. In any case, future sampling efforts in North Africa should particularly focus on these areas.

The projection of the climatic model to southwestern Europe and Mediterranean islands (fig. 5) reveals that European populations of $T$. graeca inhabit areas inside the climatic range of the species in North Africa. Although most of the non-African populations are spatially constrained to small regions, with the population in southeastern Spain being the largest (occupying approx. $2500 \mathrm{~km}^{2}$; Anadón et al., 2007, 2010), our results suggest that European populations may not be climatically constrained, at least considering the environmental factors determining the distribution in North Africa at a large scale (mainly rainfall).

Species distribution models represent simplifications of a complex ecological process (species distribution) and thus their predictions often contain errors due to deficiencies in the data and model mis-specification (e.g., lack of 
true absence data or missing covariates, Barry and Elith, 2006). In our case, we have incorporated some of the sources of uncertainty by controlling data clustering at two scales and by averaging 100 model replicates into a consensus prediction. In particular, our strategies to deal with both small and large scale data clustering largely controlled for these two sources of bias, as revealed by the comparison between final maps (e.g., fig. 4) to previous modelling trials with the original non-treated data (not shown here, see Methods). Nevertheless, as with other modelling approaches, our results should be interpreted cautiously (e.g. taking into account the underlying assumptions). This is especially true when a species niche is projected at areas outside the study region (e.g., our projection in southern Europe) and when only general environmental variables are used for the model, as this may not describe the actual niche of the species (Rödder and Lötters, 2009). Furthermore, environmental limiting situations or factors could exist in southern Europe that do not occur in North Africa, and which, therefore, cannot be captured by any model, no matter which variables are employed (Rödder and Lötters, 2009). As discussed above, this can be the case with respect to extreme minimum temperatures, that in North Africa are likely to act as a limiting factor only in a few localities (i.e., High Atlas) and thus cannot be properly captured by niche models. It should also be noted that the employed resolution $\left(5^{\prime}\right.$, about $10 \times 10 \mathrm{~km}$ ) may be adequate to capture large scale climatic patterns, but may be too coarse to describe finer climatic responses occurring at small spatial scales (i.e. steep elevational gradients).

North African populations of T. graeca are considered as threatened (IUCN, 2002), even though the true conservation status is not well known (but see Pleguezuelos et al., 2012). Our present paper delivers a starting point for a better understanding of the extant situation and future development. Much basic research is still to be done. In particular, as discussed above, models including other environmental factors (i.e., relief, land uses) acting at a finer grain (i.e. cell size) will yield more detailed results. In the same vein, deciphering possible ecological differences among the five currently recognized western Mediterranean subspecies of T. graeca (Fritz et al., 2009) could refine the present results. Finally, data describing the changes in the distribution and abundance patterns along the last decades could be employed to address the two main threats for the spur-thighed tortoise, namely, changes of land use and collection for the pet trade (e.g., Anadón et al., 2009, 2010).

Acknowledgements. This work is dedicated to the memory of Michael M.K. Lambert, who pioneered the scientific studies of the ecology and conservation of Testudo graeca in North Africa, and on whose field data this work is partially based. The authors would also like to thank to all those people that has collaborated in the collection of the field data. This work has been partially funded by the project CICYT CGL2009-08251 and the Spanish Agency for International Cooperation (AECI). J.D. Anadón was funded by a scholarship from the Fundación Seneca. J. Senko kindly reviewed the text.

\section{References}

Álvarez, Y., Mateo, J.A., Andreu, A.C., Díaz-Paniagua, C., Díez, A., Bautista, J.M. (2000): Mitochondrial DNA haplotyping of Testudo graeca on both continental sides of the Straits of Gibraltar. J. Hered. 91: 39-41.

Anadón, J.D., Gimenez, A., Martinez, M., Esteve, M.A., Pérez, I. (2006): Factors determining the distribution of the spur-thighed tortoise Testudo graeca in south-east Spain: a hierarchical approach. Ecography 29: 339-346.

Anadón, J.D., Giménez, A., Martínez, M., Esteve, M.A., Palazón, J.A. (2007): Assessing changes in habitat quality due to land use changes in Testudo graeca using hierarchical predictive habitat models. Divers. Distrib. 13: 324-331.

Anadón, J.D., Giménez, A., Ballestar, R., Perez, I. (2009): Evaluation of local ecological knowledge as a method for collecting extensive animal abundance data. Conserv. Biol. 23: 617-625.

Anadón, J.D., Giménez, A., Ballestar, R. (2010): Linking local ecological knowledge and habitat modelling to predict absolute species abundance at large scales. Biodivers. Conserv. 19: 1443-1454.

Barry, S.C., Elith, J. (2006): Error and uncertainty in habitat models. J. Appl. Ecol. 43: 413-423.

Bons, J., Geniez, P. (1996): Amphibiens et reptiles du Maroc (Sahara Occidental compris). Atlas biogéographique. Asociación Herpetológica Española, Barcelona. 
Brito, J.C., Acosta, A.L., Álvares, F., Cuzin, F. (2009): Biogeography and conservation of taxa from remote regions: An application of ecological-niche based models and GIS to North-African canids. Biol. Conserv. 142: 3020-3029.

Buckley, L.B., Rodda, G.H., Jetz, W. (2008): Thermal and energetic constraints on ectotherm abundance: a global test using lizards. Ecology 89: 48-55.

Cherkaoui, I., Selmi, S., Boukhriss, J., Hamid, R.I., Mohammed, D. (2009): Factors affecting bird richness in a fragmented cork oak forest in Morocco. Acta Oecol. 35: 197-205.

Daget, P. (1977): Mediterranean bioclimate - general characteristics and modes of definition. Vegetatio 34: 1-20.

Dormann, C.F., Mcpherson, J.M., Arau, M.B., Bivand, R., Bolliger, J., Carl, G., Davies, R.G. et al. (2007): Methods to account for spatial autocorrelation in the analysis of species distributional data: a review. Ecography 30: 609628.

Elith, J., Graham, C.H., Anderson, R.P., Dudík, M., Ferrier, S., Guisan, A., Hijmans, R.J., Huettmann, F., Leathwick, J.R., Lehmann, A., Li, J., Lohmann, L.G., Loiselle, B.A., Manion, G., Moritz, C., Nakamura, M., Nakazawa, Y., Overton, J.McC., Peterson, A.T., Phillips, S.J., Richardson, K.S., Scachetti-Pereira, R., Schapire, R.E., Soberón, J., Williams, S., Wisz, M.S., Zimmermann, N.E. (2006): Novel methods improve prediction of species' distributions from occurrence data. Ecography 29: 129-151.

Elith, J., Kearney, M., Phillips, S. (2010): The art of modelling range-shifting species. Met. Ecol. Evol. 1: 330342.

Elith, J., Phillips, S.J., Hastie, T., Dudík, M., Chee, Y.E., Yates, C.J. (2011): A statistical explanation of MaxEnt for ecologists. Divers. Distrib. 17: 43-57.

Emberger, L. (1930): Sur une formule applicable en géographie botanique. Cah. Herb. Seanc. Acad. Sci. 191: 389390.

Fritz, U., Hundsdörfer, A.K., Široký, P., Auer, M., Kami, H., Lehmann, J., Mazanaeva, L.F., Türkozan, O., Wink, M. (2007): Phenotypic plasticity leads to incongruence between morphology-based taxonomy and genetic differentiation in western Palaearctic tortoises (Testudo graeca complex: Testudines, Testudinidae). Amph-Rept. 28: 97-121.

Fritz, U., Harris, J.D., Fahd, S., Rouag, R., Graciá, E., Giménez, A., Siroky, P., Kalboussi, M., Hundsdörfer, A. (2009): Mitochondrial phylogeography of Testudo graeca in the Western Mediterranean: Old complex divergence in North Africa and recent arrival in Europe. Amphibia-Reptilia 30: 63-80.

Graciá, E., Giménez, A., Anadón, J.D., Botella, F., GarcíaMartínez, S., Marín, M. (2011): Genetic patterns of a range expansion: the spur-thighed tortoise Testudo graeca graeca in Southeastern Spain. Amph-Rept. 32: 49-61.

Guisan, A., Hofer, U. (2003): Predicting reptile distributions at the mesoscale: relation to climate and topography. Journal of Biogeography 30: 1233-1243.
Guisan, A., Thuiller, W. (2005): Predicting species distribution: offering more than simple habitat models. Ecol. Lett. 8: 993-1009.

Hijmans, R.J., Cameron, S.E., Parra, J.L., Jones, P.G., Jarvis, A. (2005): Very high resolution interpolated climate surfaces for global land areas. Int. J. Climat. 25: 1965-1978.

IUCN/Species Survival Commission (2002): IUCN Red List of Threatened Species. Gland, Switzerland.

Jiménez-Valverde, A., Barve, N., Lira-Noriega, A., Maher, S.P., Nakazawa, Y., Papeş, M., Soberón, J., Sukumaran, J. and Peterson, A.T. (2011): Dominant climate influences on North American bird distributions. Global Ecol. Biog. 20: 114-118.

Kearney, M., Porter, W.P. (2004): Mapping the fundamental niche: physiology, climate, and the distribution of a nocturnal lizard. Ecology 85: 3119-3131.

Lambert, M.R.K. (1979): Trade and the Mediterranean tortoises. Oryx 15: 81-82.

Lambert, M.R.K. (1983): Some factors influencing the Moroccan distribution of the western Mediterranean spurthighed tortoise, Testudo graeca graeca L. and those precluding its survival in NW Europe. Zool. J. Linn. Soc. 79: 149-179.

Liu, C., Berry, P.M., Dawson, T.P., Pearson, R.G. (2005): Selecting thresholds of occurrence in the prediction of species distributions. Ecography 28: 385-393.

Manel, S., Williams, H.C., Ormerod, S.J. (2001): Evaluating presence-absence models in ecology: the need to account for prevalence. J. Appl. Ecol. 38: 921-931.

Pérez, I., Giménez, A., Sánchez-Zapata, J.A., Anadón, J.D., Martínez, M., Esteve, M.A. (2004): Noncommercial extraction of a threatened species: a cultural problem of Testudo graeca graeca in Southeastern Spain. Biol. Conserv. 118: 175-181.

Pérez, I., Giménez, A., Pedreño, A. (2011): A qualitative examination of the social practices and representations towards a species of endangered tortoise. Wildl. Res. 38: 323-329.

Peterson, A.T., Papes, M., Eaton, M. (2007): Transferability and model evaluation in ecological niche modeling: a comparison of GARP and Maxent. Ecography 30: 550560.

Phillips, S.J., Dudik, M. (2008): Modeling of species distributions with Maxent: new extensions and a comprehensive evaluation. Ecography 31: 161-175.

Phillips, S.J., Anderson, R.P., Schapire, R.D. (2006): Maximum entropy modelling of species geographic distributions. Ecol. Model. 190: 231-259.

Pleguezuelos, J.M., Brito, J.C., Fahd, S., Feriche, M., Mateo, J.A., Moreno-Rueda, G., Reques, R., Santos, X. (2010): Setting conservation priorities for the Moroccan herpetofauna: the utility of Regional Red Listing. Oryx 44: 501-508.

Rhodes, J.R., McAlpine, C.A., Zuur, A.F., Smith, G.M., Ieno, E.N. (2009): GLMM applied on the spatial distribution of koalas in a fragmented landscape. In: Mixed Effects Models and Extensions in Ecology with R, p. 469-492. Zuur, A.F., Ieno, E.N., Walker, N.J., Saveliev, A.A., Smith, G.M., Eds, Springer, New York. 
Rödder, D., Lötters, S. (2009): Niche shift versus niche conservatism? Climatic characteristics of the native and invasive ranges of the Mediterranean house gecko (Hemidactylus turcicus). Glob. Ecol. Biogeogr. 18: 674-687.

Rödder, D., Schmidtlein, S., Veith, M., \& Lötters, S. (2009): Alien invasive slider turtle in unpredicted habitat: a matter of niche shift or of predictors studied? PloS ONE 4: e7843.

Salinas, M., Altet, L., Clavel, C., Almela, R.M., Bayón, A., Burguete, I., Sánchez, A. (2011): Genetic assessment, illegal trafficking and management of the Mediterranean spur-thighed tortoise in Southern Spain and Northern Africa. Conserv. Genet. 12: 1-13.

Schleich, H.H., Kästle, W., Kabisch, K. (1996): Amphibians and Reptiles of North Africa. Koeltz Scientific Books, Koenigstein.
Svenning, J.-C., Normand, S., Kageyama, M. (2008): Glacial refugia of temperate trees in Europe: insights from species distribution modelling. J. Ecol. 96: 11171127.

Vamberger, M., Corti, C., Stuckas, H., Fritz, U. (2011): Is the imperilled spur-thighed tortoise (Testudo graeca) native in Sardinia? Implications from population genetics and for conservation. Amphibia-Reptilia 32: 9-25.

Received: November 8, 2011. Accepted: April 18, 2012. 